MAGNETIC FIELD CALCULATIONS

FOR A LARGE APERTURE NARROW QUADRUPOLE

BNL/SNS TECHNICAL NOTE

NO. 121

N. Tsoupas, J. Jackson, Y.Y. Lee, D. Raparia, J. Wei BNL, Upton, NY 11973, USA

October 13, 2003

COLLIDER-ACCELERATOR DEPARTMENT

BROOKHAVEN NATIONAL LABORATORY

UPTON, NEW YORK 11973 


\title{
MAGNETIC FIELD CALCULATIONS FOR A LARGE APERTURE NARROW QUADRUPOLE*
}

\author{
N. Tsoupas, J. Jackson, Y.Y. Lee, D. Raparia, J. Wei, BNL, Upton, NY 11973 USA \\ Abstract
}

In the design of high -intensity proton synchrotrons and accumulator rings, quadrupole magnets of narrower size in one of the transverse dimensions are often needed to accommodate the compact ring geometry, the various injection and extraction devices, and the large vacuum chamber aperture. The stringent limit on tolerable beam loss further demands a good magnetic field quality to minimize beam resonances caused by higher-order magnetic multipoles.

In this paper, we present results from magnetic field calculations performed on 2D and 3D models of a large-aperture narrow-quadrupoles that is suitable for a high intensity, low beam-loss accumulator rings. The pole face of the quadrupole has been optimized to minimize the integrated field of the first three allowed multipoles (12pole,20pole and 28 pole). The ratio of each integrated magnetic-multipole-strength to the integrated magnetic-quadrupole-strength at a radius of $85 \%$ of the quadrupole's pole-tip-radius is less than $2 \times 10^{-4}$. Results from the calculations performed on the two-dimensional and three-dimensional models of the narrow quad are presented.

\section{INTRODUCTION}

In a published paper[1] we provide detailed information about the design of a "narrow quadrupole" that has been built to be used in the SNS accumulator ring[2]. The transverse dimension of the quadrupole on the horizontal plane has helped accommodate the various devices which are located at the injection and extraction regions[1] of the SNS accumulator ring. The main requirements for the magnetic design of the narrow quadrupole was to minimize the integrated strength of the 12 pole magnetic multipole down to $\mathbb{B}_{12 \text { pole }} \mathrm{dz} / \mathbb{B}_{4 \text { pole }} \mathrm{dz}<2 \times 10^{-4}$ at a radius of $\mathrm{r}=10 \mathrm{~cm}$, and we did not regard the contributions from the 20pole and 28pole multipoles. Table I shows the integrated strength of these multipoles relative to the integrated quadrupole strength as were calculated by the 3-Dimentional model and also as were measured in the first built quadrupole.

In Table $1, R_{n}=\mathbb{B}_{n}(r, z) d z / B_{1}(r, z) d z$ where $B_{n}(r, z)$ is the $n^{\text {th }}$ coefficient in the expansion of the radial field $B_{r}(r, z)$ $\mathrm{B}_{\mathrm{r}}(\mathrm{r}, \mathrm{z})=$ ? $\mathrm{B}_{\mathrm{n}}(\mathrm{r}, \mathrm{z}) \cos [(\mathrm{n}+1) \theta] \quad\{\mathrm{n}=1$ quad, $\mathrm{n}=3$ oct $\ldots\}(1)$ The $\mathrm{B}_{\mathrm{r}}(\mathrm{r}, \mathrm{z})$ is calculated and measured at a radius $\mathrm{r}=10 \mathrm{~cm}$ and the coefficients $\mathrm{B}_{\mathrm{n}}(\mathrm{r}, \mathrm{z})$ are integrated along the $\mathrm{z}$-axis which is along the beam direction.

In Table 1 , the $2^{\text {nd }}$ row shows the calculated quantity $R_{n}$, and the $3^{\text {rd }}$ and $4^{\text {th }}$ rows the measured quantity $R_{n}$ for the quadrupole as it was delivered by the manufacturer $\left(3^{\text {rd }}\right.$ row $)$ and with a minor modification (reduce the transverse pole length by $0.75 \mathrm{~mm}),\left(4^{\text {th }}\right.$ row $)$. The modification resulted in reduction of the $\mathrm{R}_{(12 \mathrm{pole})}$ multipole strength down to $1.2 \times 10^{-4}$. The error in the measurements of the harmonics was $\pm 0.02 \times 10^{-4}$.

Table 1: The Ratio $R_{n}$ of few allowed multipoles. The quantity $R_{n}$ is defined in the text.

\begin{tabular}{|l|l|l|l|} 
& $\mathrm{R}_{5(12 \mathrm{pole})}$ & $\mathrm{R}_{9(20 \text { pole })}$ & $\mathrm{R}_{13(28 \text { pole })}$ \\
\hline CALC & $-4.2 \times 10^{-4}$ & $-5.2 \times 10^{-4}$ & $-1.5 \times 10^{-4}$ \\
\hline MEAS\#1 & $+3.20 \times 10^{-4}$ & $-6.90 \times 10^{-4}$ & $-1.20 \times 10^{4}$ \\
\hline MEAS\#2 & $+1.20 \times 10^{-4}$ & $-6.81 \times 10^{4}$ & $-0.92 \times 10^{4}$ \\
\hline
\end{tabular}

Calculations performed on the SNS ring [3] showed that the measured strength of the (20 and 28)poles multipoles (see Table 1) of the narrow quadrupole are well below the limits that may bring the beam into resonance and cause significant beam emittance growth that will result in beam losses. Nevertheless we thought as a useful task to design a narrow quadrupole that minimizes the first three allowed multipoles $(12,20,28)$ pole. The following sections are dealing with the design of such a narrow quadrupole.

\section{THEORY FOR THE MAGNET DESIGN} can be expressed as:

Poisson's theorem states that the magnetic field vector B (or any vector, which is regular at infinity)

$$
\mathbf{B}(\mathbf{x})=(1 / 4 \mathrm{p}) ?\left\{\left[\nabla^{\prime}(\nabla \cdot \mathbf{B})-\nabla^{\prime} \mathrm{x}\left(\nabla^{\prime} \mathrm{x} \mathbf{B}\right)\right] /\left|\mathbf{x}-\mathbf{x}^{\prime}\right|\right\} \mathrm{d}^{3} \mathbf{x}^{\prime}
$$


By defining the magnetization vector $\mathbf{M}=\mathbf{B}-\mu_{0} \mathbf{H}$, and inserting the Maxell equations: $\nabla^{\prime} \cdot \mathbf{B}=0$ and $\nabla^{\prime} \times \mathbf{H}=\mathbf{J}$ in equation (1) we obtain eq. (2)

$$
\mathbf{B}(\mathbf{x})=(\mu 0 / 4 p)\left\{?\left[\nabla^{\prime} \mathbf{x} \mathbf{J}\right] /\left|\mathbf{x}-\mathbf{x}^{\prime}\right|\right\} \mathrm{d}^{3} \mathbf{x}^{\prime}+(1 / 4 p)\left\{?\left[\nabla^{\prime} \mathbf{x}\left(\nabla^{\prime} \mathbf{x} \mathbf{M}\right)\right] /\left|\mathbf{x}-\mathbf{x}^{\prime}\right|\right\} \mathrm{d}^{3} \mathbf{x}^{\prime}(2)
$$

Equation (2) expresses the magnetic flux density $\mathbf{B}(\mathbf{x})$ as the contribution of two terms; one term corresponding to the currents distribution $\mathbf{J}$ the other term to the magnetization $\mathbf{M}$ of the materials.

Using: $\mathbf{J}_{\mathrm{M}}=\left(1 / \mu_{0}\right)\left(\nabla^{\prime} \mathbf{x} \mathbf{M}\right)$ equation (2) becomes:

$\mathbf{B}(\mathbf{x})=\left(\mu_{0} / 4 \mathrm{p}\right)\left\{?\left[\nabla^{\prime} \mathbf{x} \mathbf{J}\right] /\left|\mathbf{x}-\mathbf{x}^{\prime}\right|\right\} \mathrm{d}^{3} \mathbf{x}^{\prime}+\left(\mu_{0} / 4 \mathrm{p}\right)\left\{?\left[\nabla^{\prime} \mathbf{x} \mathbf{J}_{\mathbf{M}}\right] /\left|\mathbf{x}-\mathbf{x}^{\prime}\right|\right\} \mathrm{d}^{3} \mathbf{x}^{\prime}$

Using the identities (4) and (5) below, in eq. (3) above, we obtain eq. (6):

$\left.\nabla^{\prime} \mathrm{x} \mathbf{J}_{\mathrm{M}}\right] /\left|\mathbf{x}-\mathbf{x}^{\prime}\right|=\nabla^{\prime} \mathrm{x}\left\{\mathbf{J}_{\mathrm{M}} /\left|\mathbf{x}-\mathbf{x}^{\prime}\right|\right\}+\mathbf{J}_{\mathrm{M}} \mathrm{x} \mathbf{r}_{\mathrm{u}} /\left|\mathbf{x}-\mathbf{x}^{\prime}\right|^{2}$

$\nabla^{\prime} \mathrm{x}\left\{\mathbf{J}_{\mathrm{M}} / \mathbf{x}-\mathbf{x}^{\prime} \mid\right\} \mathrm{d}^{3} \mathbf{x}^{\prime}=-\left\{\mathbf{J}_{\mathrm{M}} /\left|\mathbf{x}-\mathbf{x}^{\prime}\right|\right] \mathrm{xd}^{2} \mathbf{x}^{\prime}$

$\left.\mathbf{B}(\mathbf{x})=\left(\mu_{0} / 4 \mathrm{p}\right)\left\{?\left[\nabla^{\prime} \mathrm{x} \mathbf{J}\right] /\left|\mathbf{x}-\mathbf{x}^{\prime}\right|\right] \mathrm{d}^{3} \mathbf{x}^{\prime}+?\left[\mathbf{J}_{\mathrm{M}} \mathrm{x} \mathbf{r}_{\mathrm{u}} /\left|\mathbf{x}-\mathbf{x}^{\prime}\right|^{2}\right] \mathrm{d}^{3} \mathrm{x}^{\prime}-? \mathbf{J}_{\mathrm{M}} /\left|\mathbf{x}-\mathbf{x}^{\prime}\right| \mathbf{x d} \mathbf{S}^{\prime}{ }_{\text {out }}\right\}$ (6)

The third integral in eq. (6) extends over the surface which encloses the whole volume of the space therefore vanishes because the $\mathbf{J}_{\mathrm{M}}$ is zero at large distances (No magnetic magnet iron).

The second integral in eq. (6) can be written as:

$\left.\left.? \mathbf{J}_{\mathrm{M}} \mathrm{x} \mathbf{r}_{\mathrm{u}} / \mid \mathbf{x}-\mathbf{x}^{\prime}{ }^{\imath}\right] \mathrm{d}^{3} \mathrm{x}^{\prime}=?\left[\mathbf{J}_{\mathrm{M}} \mathrm{x} \mathbf{r}_{\mathrm{u}} / \mid \mathbf{x}-\mathbf{x}^{\prime}{ }^{\natural}\right] \mathrm{d}^{3} \mathrm{x}^{\prime}+? \mathbf{J}_{\mathrm{M}^{\mathrm{x}}} \mathbf{r}_{\mathrm{u}} /\left|\mathbf{x}-\mathbf{x}^{\prime}\right|^{2}\right] \mathrm{d}^{3} \mathrm{x}^{\prime} \quad$ (7)

Inner Volume Volume of boundary layer

The integral over the volume of the "boundary layer" of the material can be written:

$$
\begin{aligned}
& \left\{\mathbf{J}_{\mathrm{M}} \mathrm{x} \mathbf{r}_{\mathrm{u}} /\left|\mathbf{x}-\mathbf{x}^{\prime}\right|^{2}\right] \mathrm{d}^{3} \mathrm{x}^{\prime}=? \nabla \mathrm{x}\left[\mathbf{J}_{\mathrm{M}} /|\mathbf{x}-\mathbf{x}|\right] \mathrm{d}^{3} \mathrm{x}^{\prime}=\nabla \mathrm{x}\left[? \mathbf{J}_{\mathrm{M}} /\left|\mathbf{x}-\mathbf{x}^{\prime}\right|\right] \mathrm{d}^{3} \mathrm{x}^{\prime}=\left\{\mathrm{Use}: \nabla[\mathrm{X} / \mathrm{r}]=\mathbf{r}_{\mathrm{u}}[\mathrm{X} / \mathrm{r}]\right\} \\
& =\left(1 / \mu_{0}\right)\left(\nabla \times \mathfrak{X}\left(\nabla^{\prime} \mathrm{x} \mathbf{M}\right) /\left|\mathbf{x}-\mathbf{x}^{\prime}\right|\right] \mathrm{d}^{3} \mathrm{x}^{\prime}=\quad \quad\left\{\text { Use: } \mathbf{J}_{\mathrm{M}}=\left(1 / \mu_{0}\right)\left(\nabla^{\prime} \mathrm{x} \mathbf{M}\right)\right\} \\
& =\left(1 / \mu_{0}\right) \nabla \mathrm{x}\left\{?\left[\nabla \mathrm{x}\left[\mathbf{M} /\left|\mathbf{x}-\mathbf{x}^{\prime}\right|\right] \mathrm{d}^{3} \mathrm{x}^{\prime}+\nabla^{\prime} \mathrm{x}\left[\mathbf{M} /\left|\mathbf{x}-\mathbf{x}^{\prime}\right|\right] \mathrm{d}^{3} \mathrm{x}^{\prime}\right\} \quad\left\{\text { Use: }\left(\nabla^{\prime} \mathbf{M}\right) /\left|\mathrm{x}-\mathrm{x}^{\prime}\right|=\nabla\left[\mathbf{M} /\left|\mathrm{x}-\mathrm{x}^{\prime}\right|\right]+\nabla^{\prime}\left[\mathbf{M} /\left|\mathrm{x}-\mathrm{x}^{\prime}\right|\right]\right\}\right. \\
& =\left(1 / \mu_{0}\right) \nabla \mathrm{x}\left\{\nabla \mathrm{x} ?\left[\mathbf{M} /\left|\mathbf{x}-\mathbf{x}^{\prime}\right|\right] \mathrm{d}^{3} \mathrm{x}^{\prime}-?\left[\mathbf{M} /\left|\mathbf{x}-\mathbf{x}^{\prime}\right|\right] \mathrm{d}^{2} \mathrm{x}^{\prime}\right\} \quad\left\{\text { Use: } \nabla^{\prime} \mathrm{x}\left[\mathbf{M} /\left|\mathbf{x}-\mathbf{x}^{\prime}\right| \mid \mathrm{d}^{3} \mathbf{x}^{\prime}=-\left\{\mathbf{M} /\left|\mathbf{x}-\mathbf{x}^{\prime}\right|\right] \mathrm{xd} \mathbf{S}^{\prime}{ }_{\text {out }}\right\}\right.
\end{aligned}
$$

The volume integral $\left.? \mathbf{M} /\left|\mathbf{x}-\mathbf{x}^{\prime}\right|\right] \mathrm{d}^{3} \mathbf{x}^{\prime}=0$ as the volume layer approaches to zero volume.

The surface integral $-\left(1 / \mu_{0}\right) \nabla \times\left[\mathbf{M} /\left|\mathbf{x}-\mathbf{x}^{\prime}\right|\right] \mathrm{d} \mathbf{S}^{\prime}{ }_{\text {out }}=-\left(1 / \mu_{0}\right) ? \mathrm{x}\left[\mathbf{M} /\left|\mathbf{x}-\mathbf{x}^{\prime}\right| \mathrm{d} \mathbf{S}_{\text {out }}^{\prime}=\right.$

$-\left(1 / \mu_{0}\right) \gtrless \times\left[1 /\left|\mathbf{x}-\mathbf{x}^{\prime}\right|\right]\left[\mathbf{M x d} \mathbf{S}_{\text {out }}^{\prime}\right]=\left(1 / \mu_{0}\right) ?\left[\mathbf{r}_{\mathrm{u}} /\left|\mathbf{x}-\mathbf{x}^{\prime}\right|^{2}\right] \mathrm{x}\left[\mathbf{M x d} \mathbf{S}_{\text {out }}^{\prime}=^{\prime}\right]=\left(1 / \mu_{0}\right)\left\{\mathbf{M x d} \mathbf{S}_{\text {out }}^{\prime} \mathbf{x} \mathbf{r}_{\mathrm{u}}\right] /\left|\mathbf{x}-\mathbf{x}^{\prime}\right|^{2}$

Thus equation (6) can be written as:

$$
\mathbf{B}(\mathbf{x})=\left(\mu_{0} / 4 \mathrm{p}\right)\left\{?\left[\nabla^{\prime} \mathrm{x} \mathbf{J}\right] /\left|\mathbf{x}-\mathbf{x}^{\prime}\right|\right] \mathrm{d}^{3} \mathbf{x}^{\prime}+\left\{\left[\mathbf{J}_{\mathrm{M}} \mathrm{x} \mathbf{r}_{\mathrm{u}} /\left|\mathbf{x}-\mathbf{x}^{\prime}\right|^{2}\right] \mathrm{d}^{3} \mathrm{x}^{\prime}+\left(1 / \mu_{0}\right)\left\{\left[\mathbf{M x d} \mathbf{S}^{\prime}{ }_{\text {out }} \mathrm{x} \mathbf{r}_{\mathrm{u}}\right] /\left|\mathbf{x}-\mathbf{x}^{\prime}\right|^{2}\right\} \quad \text { (8) } \quad\left(\mathbf{r}_{\mathrm{u}}=\text { unit vector along } \mathbf{x}-\mathbf{x}^{\prime}\right)\right.
$$

In equation (8) the second integral extends over the interior of the finite volume of the magnetic material, and the third integral over the surface enclosing the volume of the magnetic material ( $\mathrm{d} \mathbf{S}^{\prime}{ }_{\text {out }}=$ normal to the surface element).

It is the contribution of the third integral that can affect the strength of the various allowed multipoles by altering the contour of the pole face. We assume that the value of the permeability $\mu$ of the iron at the vicinity of the pole surface has a value $\mu>>1$ for the third integral to have an effect on the magnetic multipoles. It is therefore possible to affect the magnetic field in the space of the beam by modifying the contour of the pole tip of the quadrupole. This approach [4] was followed in the design of narrow quadrupoles. In this paper we employ more sophisticated contour of the pole tip and we extend the calculations in three dimensions. 


\section{TWO-DIMENSIONAL MODELING}

In this section we present the results of the two dimensional magnetic calculations as applied to three design $\mathrm{s}$ of the narrow quadrupole. The three designs will be referred in the text as A,B and C. In each of the designs, discussed below, we provide enough information about the cross section of the narrow quadrupoles, for the reader to repeat the calculations using her/his preferred computer code for the elelectomagnetic design. In all three designs the strength of all allowed multipoles $\mathrm{B}_{(12 \mathrm{pole}, 20 \mathrm{pole}, 28 \mathrm{pole})}$ was calculated at $\mathrm{r}=10 \mathrm{~cm}$ and the $\mathrm{B}_{(12 \mathrm{pole}, 20 \mathrm{pole}, 28 \mathrm{pole})} / \mathrm{B}_{\text {quad }}$ ratio was reduced below the value of $1 \times 10^{-4}$. The deviation of the narrow quad from the four fold symmetry, has introduced multipoles like octupoles, 16 poles etc. However the relative strength of each of the multipoles $\left.\left(\mathrm{B}_{(8 \text { pole, } 16 \text { pole,2qole }}\right) \mathrm{B}_{\text {quad }}\right)$ was below the value of $1 \times 10^{-5}$ at $\mathrm{r}=10 \mathrm{~cm}$. In each of the designs we kept intact both, the outside dimensions of the quadrupole (shown in Fig. 1) and the pole tip radius $\mathrm{R}_{\mathrm{p}}$ and we only varied the width of the pole piece $\mathrm{W}$, and the pole tip contour. In order to keep the permeability of the iron at a reasonable large value $\mu \gg>1$, the quadrupole strength of each of the models was also kept at a value of $\sim 4.2[\mathrm{~T} / \mathrm{m}]$. All calculations were performed using the computer code for electomagnetics of Vector_Fields[5].

\subsection{Narrow Quadrupole Design_A}

The cross section of one of the pole pieces of the desisign_A quadrupole is shown in Fig 2. In this design we kept the contour of the pole tip similar to the contour of the narrow quad discussed in ref [1] but we increased the pole width (W) to a value of $19.8 \mathrm{~cm}$, to achieve minimization of the 20pole and 28pole multipoles. The design was finally optimized by modifying the contour of the pole face, by varying the radii of curvature $?_{\mathrm{i}}, ?_{\mathrm{o}}$ and the location of the inflection points $\mathrm{P}_{1}, \mathrm{P}_{2}$, shown in Fig 2 .

The optimization yielded a ratio $B_{n} / B_{\text {quad }}$ of $<1 \times 10^{-4}$ at a radius $r=10 \mathrm{~cm}$ for the $(12,20,28)$ pole multipoles . The increase of the pole width (W) however reduced the area of the current conductor which has to run at a higher current density $(\mathrm{J})$ to achieve the quadrupole strength of $\sim 4.2[\mathrm{~T} / \mathrm{m}]$. An alternative design which satisfies the requirements of low relative strength $\mathrm{B}_{\text {npole }} / \mathrm{B}_{4 \text { pole }}<1 \times 10^{-4}$, for the $(12,20,28)$ pole multipoles, and also provides more conductor area, is discussed in the next subsection.

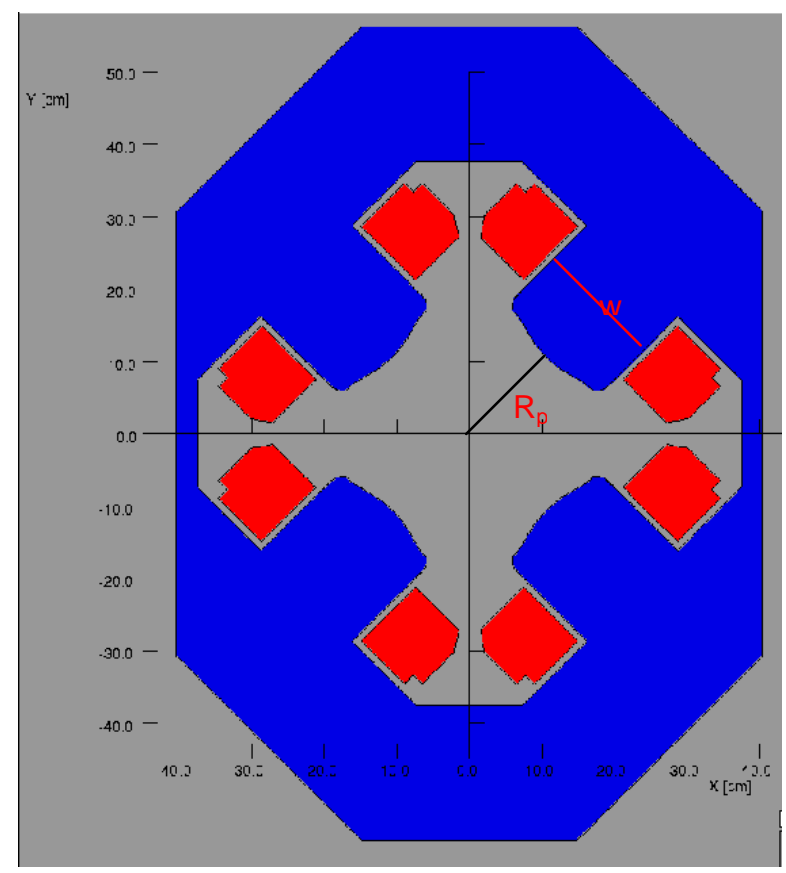

Figure 1: Cross section of the narrow quad. The outer dimensions were the same for all designs A,B, and C.

\subsection{Narrow Quadrupole Design_B}


The cross section of this alternative design of a narrow quadrupole is shown in Fig. 3. In this design the width of the pole piece has been reduced to $17.6 \mathrm{~cm}$ but the overall shape of the pole tip surface remained almost the same as in design_A with only small modifications of the location of the inflection points $\mathrm{P}_{1}, \mathrm{P}_{2}$ and radii of curvature ?i, ? ${ }_{0}$. These minor modifications reduced the relative strength $\mathrm{B}_{\text {npole }} / \mathrm{B}_{4 \text { pole }}$ of the $(12,20,28)$ pole multipoles, below the required value of $1 \times 10^{-4}$ at a radius $\mathrm{r}=10 \mathrm{~cm}$.

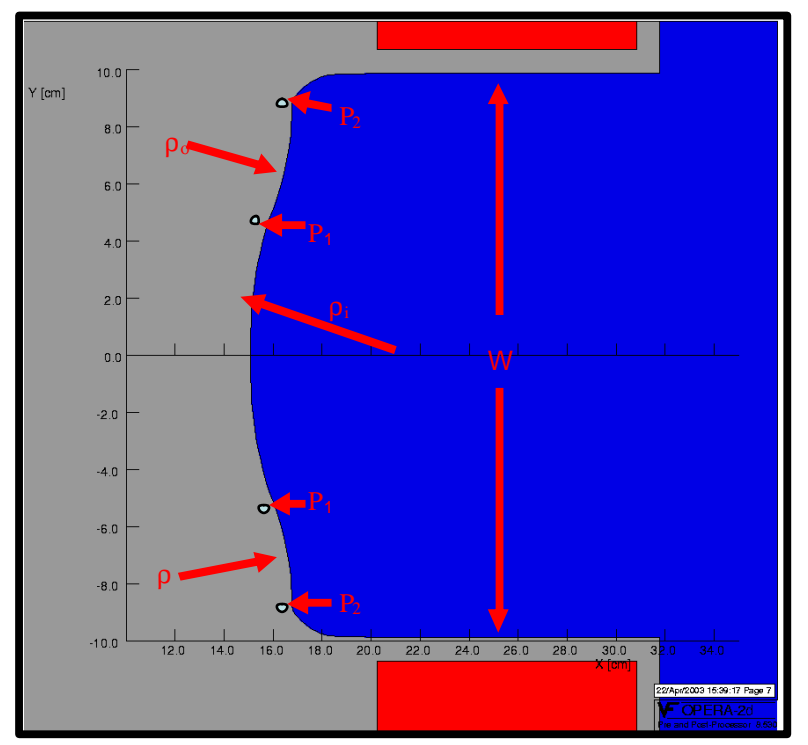

Figure 2. Cross section of pole piece corresponding to "design A" (see text). The inflection points $\mathrm{P}_{1}, \mathrm{P}_{2}$, and the radii of curvature $\rho_{i}, \rho_{\mathrm{o}}$ were varied in order to minimize the strength of the $(12,20,28)$ pole multipoles .

Compared with design A, this design allows for an increased cupper area and the required gradient of $\sim 4.2$ [T/m] is achieved at a reduced current density, but the magnet ic field $\mathbf{B}$ inside the poles will be higher for the same value of the magnetic field at the pole tip.

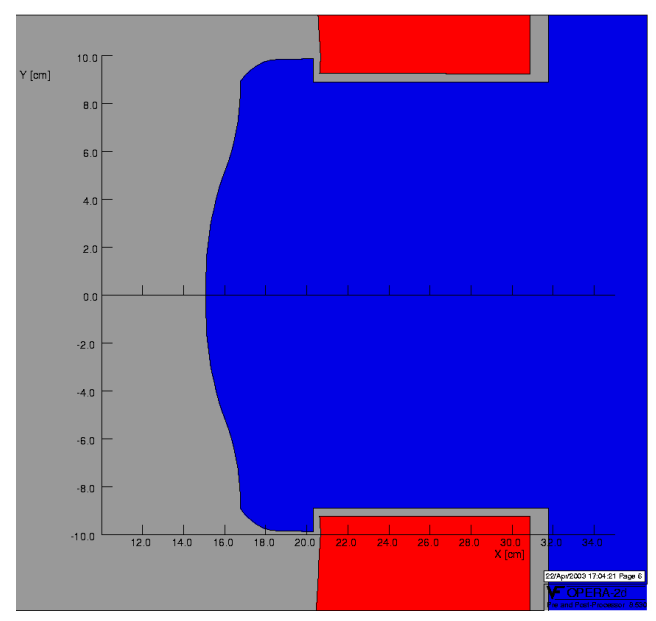

Figure 3. Cross section of pole piece for "design B" .

\subsection{Narrow Quadrupole Design_C}

This design combines the features of the design_A and design_B namely larger conductor area (same as in design_B) and lower value of the magnetic flux density $\mathbf{B}$ inside the pole pieces (as in design_A). The cross section is shown in 
figure 4. Tthe relative strength $B_{\text {npole }} / B_{4 \text { pole }}$ of the $(12,20,28)$ pole is minimized to values $<1 \times 10^{-4}$. Compare the contour shape of design_C with that of designs_A or B.

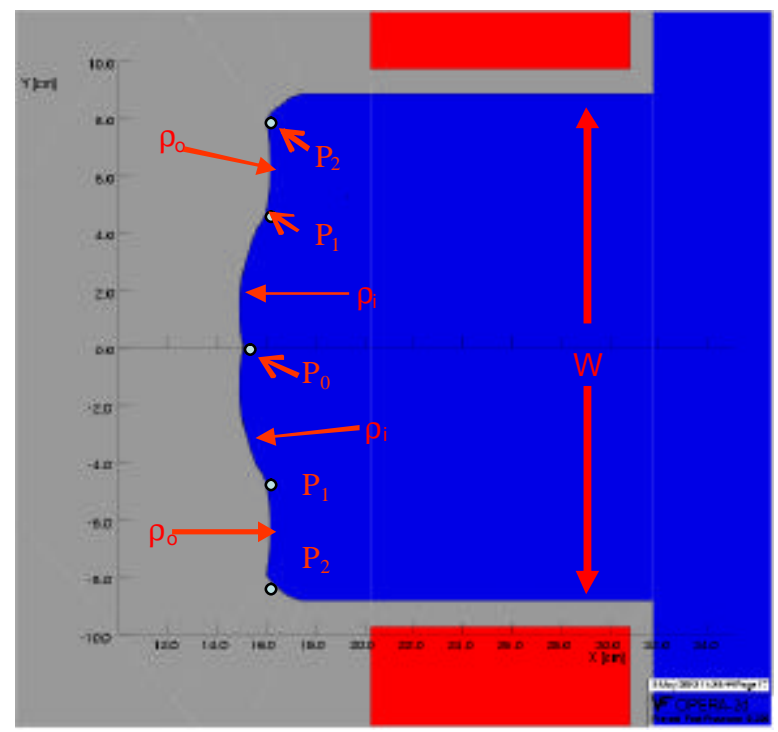

Figure 4. Cross section of the pole piece of "design_C".

\section{THREE-DIMENSIONAL MODELING}

Practical considerations lead us to perform the three dimensional magnetic field calculations on the "design_C". The goal was to minimize the relative integrated strength $\mathbb{B}_{\text {npole }} \mathrm{dz} / \mathbb{B}_{4 \text { pole }} \mathrm{dz}$ of the $(12,20,28)$ pole multipoles . The method of optimization was to chamfer the edges of the pole pieces at both the entrance and exit of the magnet [1] as shown in figure 6. The "pole chamfering" reduced the integrated strength of the 12pole multipole but introduced some strength in the 20pole and 28pole multipoles. This strength was reduced by reshaping slightly the contour of the pole tip inside the magnet. The optimization yielded the following results:

$\mathbf{B}_{12 \text { pole }} \mathrm{dz} / \mathbf{B}_{4 \text { pole }} \mathrm{dz}=2 \times 10^{-6} \quad \mathbf{B}_{\text {20pole }} \mathrm{dz} / \mathbf{B}_{4 \text { pole }} \mathrm{dz}=4 \times 10^{-5} \mathbf{B}_{28 \text { pole }} \mathrm{dz} / \mathbf{B}_{4 \text { pole }} \mathrm{dz}=5 \times 10^{-5}$ at $\mathrm{r}=10 \mathrm{~cm}$.

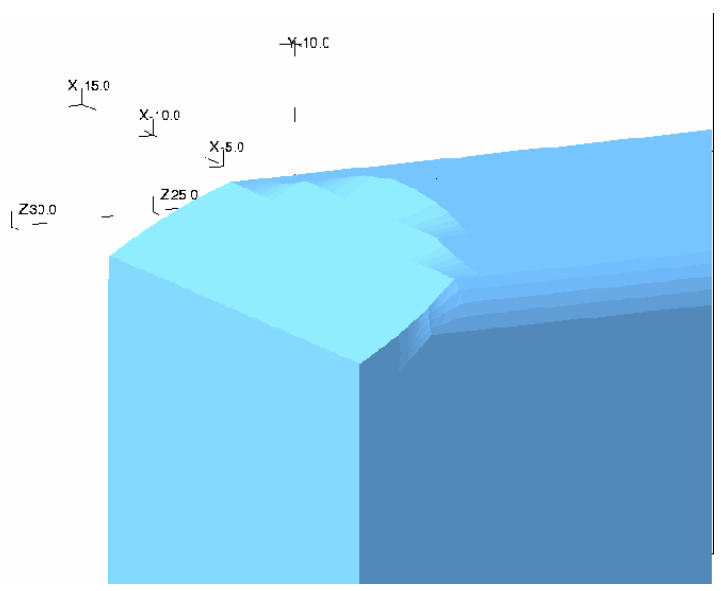

Figure $5 \mathrm{An}$ isometric view of the chamfered ends of one of the pole-piece of the narrow quadrupole. 


\section{CONCLUSIONS}

Two dimensional magnetic field calculations were performed on three models of a large aperture narrow quadrupole. Each of the models was optimized to minimize the relative strength $\mathrm{B}_{\text {npole }} / \mathrm{B}_{4 \text { pole }}$ of the $(12,20,28)$ pole multipoles to values less than $1 \times 10^{-4}$ at a radius $\mathrm{r}=10 \mathrm{~cm}$. One of the models was optimized using $3 \mathrm{D}$ magnetic field calculations.

\section{REFERENCES}

[1] N. Tsoupas, et. al. "Large Aperture Narrow Quadrupole for the SNS Accumulator Ring"Proc. EPAC2002 p. 1106 Paris June 3-7 2002

[2] J. Wei, et. al. Phys. Rev. ST AB 3, 080101 (2000)

[3] G.Parzen, A. Fedotov, BNL Private Communication

[4] G.T Danby and J. Jackson "Theory Design and Measurements of the Brookhaven Narrow Quadrupoles"

Proceedings National Particle Accelerator Conf. Wash. DC March 1-3 1967

[5] Vector Fields Inc. 